\title{
Intra-cellular traffic: bio-molecular motors on filamentary tracks*
}

\author{
Debashish Chowdhury ${ }^{\dagger},{ }^{1}$ Aakash Basu, ${ }^{1}$ Ashok Garai, ${ }^{1}$ Philip Greulich,${ }^{2}$ \\ Katsuhiro Nishinari, ${ }^{3}$ Andreas Schadschneider, ${ }^{2}$ and Tripti Tripathi ${ }^{1}$ \\ ${ }^{1}$ Department of Physics, Indian Institute of Technology, Kanpur 208016, India \\ ${ }^{2}$ Institute for Theoretical Physics, University of Cologne, D-50937 Köln, Germany \\ 3 Department of Aeronautics and Astronautics, Faculty of Engineering, \\ University of Tokyo, Hongo, Bunkyo-ku, Tokyo 113-8656, Japan
}

(Dated: October 26, 2018)

\begin{abstract}
Molecular motors are macromolecular complexes which use some form of input energy to perform mechanical work. The filamentary tracks, on which these motors move, are made of either proteins (e.g., microtubules) or nucleic acids (DNA or RNA). Often, many such motors move simultaneously on the same track and their collective properties have superficial similarities with vehicular traffic on highways. The models we have developed provide "unified" description: in the low-density limit, a model captures the transport properties of a single motor while, at higher densities the same model accounts for the collective spatio-temporal organization of interacting motors. By drawing analogy with vehicular traffic, we have introduced novel quantities for characterizing the nature of the spatio-temporal organization of molecular motors on their tracks. We show how the traffic-like intracellular collective phenomena depend on the mechano-chemistry of the corresponding individual motors.
\end{abstract}

PACS numbers: 89.20.-a;89.75.-k

\section{INTRODUCTION}

Motility is the hallmark of life. A distinguishing feature of molecular motor transport in eukaryotic cells is that the motor proteins move on filamentary "tracks" [1, 2, 3, 4]. The tracks for motor proteins are made of either proteins or nucleic acids. Not all motor proteins carry molecular cargo. A common feature of all these motors is that these perform mechanical work by utilizing some other form of input energy and hence the name "motor". All the molecular motors we consider in this paper directly convert chemical energy into mechanical work.

During several biological processes many motors move simultaneously on the same track. The collective movement of the motors under such cicumstances strongly resemble vehicular traffic flow [5, 6]. In this paper we present few models of molecular motor traffic which, unlike earlier works of other groups, also capture the essential steps of the mechano-chemistry of individual motors.

Our models of molecular motor traffic are biologically motivated extensions of the totally asymmetric simple exclusion process (TASEP) [7]. A TASEP is defined in terms of "rules" for updating the states of the system: a particle can move forward (with probability $q$ ) by one lattice specing if, and only if, the target site is empty. Updating can be implemented either in parallel or in random-sequential manner; properties of the model depends on the updating scheme. For a finite system, either

* Based on the invited talk delivered by DC at the IUPAP International Conference STATPHYS23, Genoa (Italy), July, 2007.

${ }^{\dagger}$ E-mail: debch@iitk.ac.in (Corresponding author) periodic boundary conditions (PBC) or open boundary conditions (OBC) can be imposed. When the boundaries are open, at every time step, a particle can enter the system (and occupy the site $j=1$ ) with probability $\alpha$, if the site $j=1$ is empty. Similarly, under OBC, a particle occupying the site $j=L$ can exit with probability $\beta$. TASEP under OBC shows an interesting phase diagram and is the prototype for so-called boundary-induced phase transitions [8]. To our knowledge, TASEP is the simplest model of a system of interacting self-propelled particles on a discrete lattice. It has been extended in several ways to formulate "particle-hopping" models for capturing various interesting aspects of vehicular traffic [9].

Our aim is to analyze molecular motor traffic from the perspective of vehicular traffic. Therefore, let us first list some of the important quantities used in traffic science for characterizing traffic flow [9]. In the "particle-hopping" models of vehicular traffic each vehicle is represented by a particle. The number of particles leaving a detector site per unit time is defined as the flux and the relation between the average flux and the number density of the particles is called the fundamental diagram.

In the particle-hopping models of vehicular traffic [9], the time interval in between the departure of successive particles from a detector site is defined as the time headway $(\mathrm{TH})$. Let us start our clock as soon as a particle (let us label it by the integer $j=0$ ) just leaves the detector whose location is fixed. Suppose, the next $n$ particles leave the same detector at times $t_{1}, t_{2}, \ldots t_{n}$, respectively. Then, the corresponding THs are $\tau_{1}=t_{1}, \tau_{2}=t_{2}-t_{1}$, $\tau_{3}=t_{3}-t_{2}, \ldots, \tau_{n}=t_{n}-t_{n-1}$, respectively. The average flux (averaged over the time interval $t_{n}$ ) is $J=n / t_{n}$. Since $\sum_{j=1}^{n} \tau_{j}=t_{n},\left(\sum_{j=1}^{n} \tau_{j}\right) / n=1 / J$, i.e., the mean 
$T H$ is the inverse of the average flux; therefore, the distribution of the THs contains more detailed informations on the flow characteristics of traffic than what is available from average flux [10, 11].

The average density-profile of the particles is yet another quantitative characteristic of TASEP and TASEPlike models. A more detailed characterization of the spatial-organization of the particles is possible in terms the distribution of the distance-headways (DHs) where $\mathrm{DH}$ between two successive particles is defined to be the number of empty sites in between them [12, 13].

In our earlier papers on models of molecular motor traffic [14, 15, 16, 17, 18, 19] we reported results on average flux under both $\mathrm{PBC}$ and $\mathrm{OBC}$ as well as the average density profiles and the phase diagrams under OBC. In a few cases [16, 19], we have also reported distributions of $\mathrm{TH}$ under OBC. In this paper, for each example of molecular motor traffic, we briefly summarize the model and the fundamental diagram before presenting new results on the distributions of THs. We also briefly discuss our new observations on the distributions of DHs in these models. The TH distributions have important biological implications and have been discussed in detail elsewhere [16, 19]. Three examples of molecular motor traffic are considered in the sections III III and IV The DH distributions in the models of molecular traffic are discussed briefly in section $\mathrm{V}$. Our summary and outlook is presented in section VI

\section{TRAFFIC OF KINESINS ON MICROTUBULE TRACK}

Microtubules and filamentous actin serve as the tracks for cytoskeletal motors and both are made of proteins [1]. The members of the kinesin and dynein superfamilies of cytoskeletal motors move on microtubules (MT) whereas those of the myosin superfamily move on actin filaments. These motors run on chemical fuel in the sense that the mechanical energy required for their movement is supplied from the energy released by the hydrolysis of adenosine triphosphate (ATP); the products of the hydrolysis reaction being adenosine diphosphate (ADP) and an inorganic phosphate [4].

Cytoskeletal motors can attach at any motor-binding site on a MT track and can also detach from the track [1]. This distinct feature of the cytoskeletal motors was captured in the older TASEP-type models of molecular motor traffic [20, 21, 22, 23, 24, 25] by adding Langmuirlike attachment and detachment processes to the updating rules of TASEP. More specifically, in those models, a particle is allowed not only to hop forward, but also to "attach" to any empty lattice site (with rate $\omega_{a}$ ), and "detach" from an occupied site (with rate $\omega_{d}$ ). In a well-defined regime of parameters, this model exhibits co-existence of high-density and a low-density regions which are separated by a domain wall (shock); this type of spatio-temporal organization is interpreted as a jam in molecular motor traffic. But, since most of the effects of each mechano-chemical cycle of a motor is captured in these models through a single effective hopping rate $q$, it is difficult to make a direct quantitative comparison with experimental data which depend on such chemical processes.

We have focussed attention on a family of singleheaded kinesins, called KIF1A. In order to develope a model for cytoskeletal motor traffic, which would not suffer from the limitations of the earlier TASEP-like models, we have incorporated the essential steps of the mechanochemical cycle of individual KIF1A motors in our model. Each biochemical cycle of a KIF1A motor consists of a sequence of four states, namely, kinesin (K), kinesin bound with ATP (KT), kinesin bound with ADP and phosphate (KDP) and, finally, kinesin bound with only ADP (KD). The motor binds strongly to the MT track in both the states K and KT; the state KDP has very short life time and KD binds weakly to the track. Therefore, at each spatial location in our simplified model, a KIF1A is allowed to exist in one of the two distinct "chemical" states depending on whether it is bound strongly or weakly to the track; these two chemical states are denoted by the symbols $S$ and $W$, respectively.

The allowed transitions and the corresponding rate constants are shown in fig 1. The rate constants $\omega_{a}$ and $\omega_{d}$ account for the attachments and detachments of the motors. The rate constant $\omega_{b}$ corresponds to the unbiased one-dimensional Brownian motion of the motor in the state where it is weakly bound to the MT track. The rate constant $\omega_{h}$ is associated with the process driven by ATP hydrolysis which causes the transition of the motor from the state $S$ to the state $W$. The rate constants $\omega_{f}$ and $\omega_{s}$, together, capture the Brownian ratchet mechanism of movement of a KIF1A motor [14, 15]. As in the earlier TASEP-type models of cytoskeletal motor traffic $[20,21,22,23,24,25]$, none of the lattice sites is allowed to be occupied by more than one motor at a time.

Let $S_{i}(t)$ and $W_{i}(t)$ denote the probabilities of finding the motor at location $i$ in the strongly- and weakly-bound states, respectively. The master equations for $S_{i}$ and $W_{i}$ are given by [14]

$$
\begin{aligned}
\frac{d S_{i}}{d t}= & \omega_{s} W_{i}+\omega_{f} W_{i-1}\left(1-S_{i}-W_{i}\right) \\
& +\omega_{a}\left(1-S_{i}-W_{i}\right)-\omega_{d} S_{i}-\omega_{h} S_{i} \\
\frac{d W_{i}}{d t}= & \omega_{h} S_{i}+\omega_{b}\left(W_{i-1}+W_{i+1}\right)\left(1-S_{i}-W_{i}\right) \\
& -\omega_{s} W_{i}-\omega_{f} W_{i}\left(1-S_{i+1}-W_{i+1}\right) \\
& -\omega_{b} W_{i}\left(2-S_{i+1}-W_{i+1}-S_{i-1}-W_{i-1}\right)
\end{aligned}
$$

Solving these equations in the steady state under PBC, we get the flux

$$
J=\left[\frac{\omega_{h}}{\omega_{h}+\omega_{s}+\omega_{f}(1-\rho)}\right] \omega_{f} \rho(1-\rho)
$$




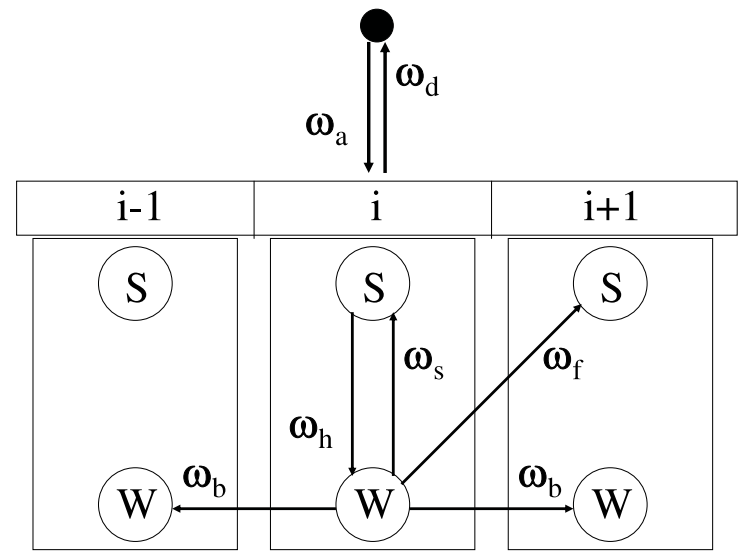

FIG. 1: Schematic description of the mechano-chemical cycle of a single-headed kinesin motor KIF1A in our model. The equispaced sites labelled by the integers $\ldots, i-1, i, i+1, \ldots$ denote the binding sites of the motor on the microtubule (MT) track. The encircled symbols $S$ and $W$ denote the two "chemical" states of the motor in which it is, respectively, strongly and weakly bound to the track. The allowed transitions are indicated by the arrows and the symbols accompanying the arrows are the corresponding rate constants.

where

$$
\rho=S+W=\frac{\Omega_{h}+\Omega_{s}+\left(\Omega_{s}+1\right) K-\sqrt{D}+2}{2(1+K)} .
$$

is the steady-state number-density of the motors on the MT track, with $K=\omega_{d} / \omega_{a}, \Omega_{h}=\omega_{h} / \omega_{f}, \Omega_{s}=\omega_{s} / \omega_{f}$, and

$$
D=4 \Omega_{s} K(1+K)+\left(\Omega_{h}+\Omega_{s}+\left(\Omega_{s}-1\right) K\right)^{2} .
$$

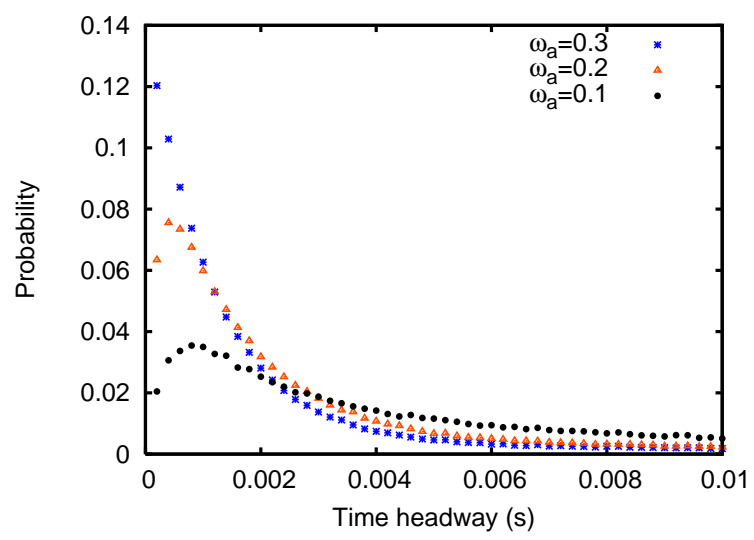

FIG. 2: TH distributions in the model of KIF1A traffic under OBC. The three curves correspond to three different values of $\omega_{a}$ (in the units of $s^{-1}$ ). The other parameters are $\omega_{d}=0.1 s^{-1}, \omega_{h}=100 s^{-1}, \omega_{s}=145 s^{-1}, \omega_{f}=55 s^{-1}$, $\omega_{b}=1125 s^{-1}, \alpha=50 s^{-1}$ and $\beta=700 s^{-1}$.

In this model, departure of a motor from a site can take place in two different ways: either by hopping to the next site or by detachment from the track. Therefore, we first modify the original definition of $\mathrm{TH}$, which we presented in the introductory section, for our model of KIF1A under OBC. We interpret the attachments at the site $i=1$ and detachments at $i=L$ as consequences solely of forward hoppings of the motors, rather than manifestations of Langmuir-like kinetics. The time interval in between the exit of two successive motors from the site $i=L$ is identified as the corresponding TH.

A few typical TH-distributions under $\mathrm{OBC}$ in our model of KIF1A traffic are plotted in fig 2 26]. A higher $\omega_{a}$ leads to a higher average density of motors on the track which, in turn, reduces the most probable $\mathrm{TH}$ as long as $\beta$ remains sufficiently high. This trend of variation is consistent with that of the average flux with $\omega_{a}$ [14, 15]. Moreover, a wider distribution at smaller $\omega_{a}$ indicates stronger fluctuations in the THs at a lower average density. In principle, these new theoretical predictions can be tested by carrying out in-vitro experiments with fluorescently-labelled KIF1A molecules using single-molecule imaging techniques. Systematic study of the variation of the width of the $\mathrm{TH}$ distribution with the control parameters of the experiment will provide deep insight into the nature of "noise" in intracellular cytoskeletal motor transport.

\section{TRAFFIC OF RNAP MOTORS ON DNA TRACKS}

Polymerization of a mRNA from the corresponding DNA template is carried out by a motor called RNA polymerase (RNAP) [27] and the process is called transcription. To our knowledge, all the models of transcription reported earlier [28, 29, 30, 31, 32, 33, 34, 35, 36, 37, 38] capture only the stochastic mechano-chemistry of the individual RNAP motors. Cooperation and collisions between RNAP motors is known to have non-trivial effects on the rate of transcription [39, 40, 41, 42]. Moreover, the possibility of the formation of queues in RNAP traffic has also been explored [43].

The interactions of RNAPs in transcriptional interference 44] is a well known phenomenon and it has also been modelled quantitatively [42]. However, instead of studying interactions of RNAPs during the transcription of different genes, we have modelled the steric interactions of RNAPs which are simultaneously involved in the transcription of the same gene.

The model is described schematically in fig 3 . Each of the lattice sites corresponds to a single nucleotide on the DNA template. Each successful addition of a nucleotide to the elongating RNA is accompanied by a forward stepping of the RNAP. A single mechano-chemical cycle of the RNAP during this elongation stage consists of several steps of which the major ones are as follows: (i) Nucleoside triphosphate (NTP) binding to the active site of the RNAP when the active site is located at the tip of the growing RNA transcript, (ii) NTP hydrolysis, (iii) re- 


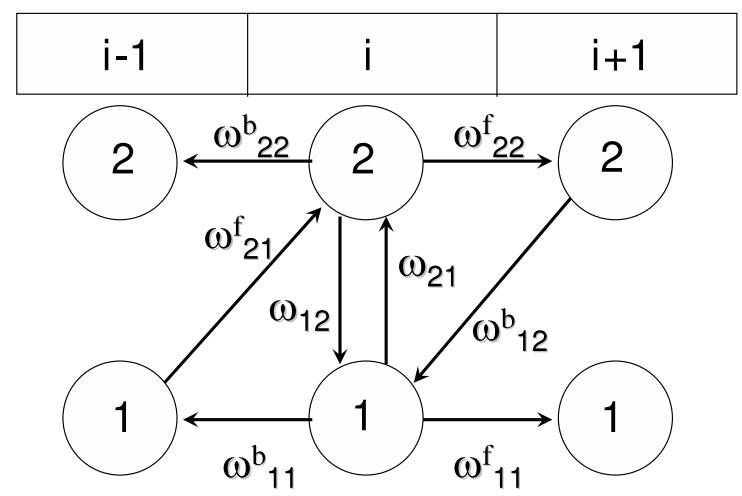

FIG. 3: A schematic representation of the mechano-chemical cycle of each RNAP in our model. The equispaced sites labelled by the integers $\ldots, i-1, i, i+1, \ldots$ denote the nucleotides on the template DNA track. No $P P_{i}$ is bound to the RNAP in the state 1 whereas the pyrophosphate $\left(P P_{i}\right)$-bound state of the RNAP is labelled by the index 2 . The allowed transitions denoted by arrows and the corresponding rate constants are also shown.

lease of pyrophosphate $\left(P P_{i}\right)$, one of the products of hydrolysis, and (iv) accompanying forward stepping of the RNAP. Since $P P_{i}$-release is known to be the rate-limiting step, we consider only two distinct chemical states $\mu$ of the RNAP; $\mu=1$ refers to the state in which the RNAP is not bound to any $P P_{i}$ whereas $\mu=2$ corresponds to the state with bound $P P_{i}$.

The processes corresponding to the rate constants $\omega_{12}$ and $\omega_{21}$ are dominated by $P P_{i}$-release and its reverse reaction. The symbol $\omega_{21}^{f}$ is the rate of the successful addition of an NTP, catalyzed by the RNAP, whereas $\omega_{12}^{b}$ is that of the reverse reaction. The remaining four rate constants correspond to polymerization/depolymerization of the RNA, by one monomer, unaided by the RNAP. Since premature detachment of a RNAP from its track is a very rare event during the elongation of the growing RNA, we do not allow such processes in our model.

The values of some of the rate constants used in our numerical studies are as follows: $\omega_{12}=31.4 \mathrm{~s}^{-1}, \omega_{12}^{b}=$ $0.21 s^{-1}, \omega_{11}^{f}=4.66 \times 10^{-5} s^{-1}, \omega_{11}^{b}=9.4 s^{-1}, \omega_{22}^{f}=$ $0.31 \times 10^{-6} s^{-1}, \omega_{22}^{b}=0.063 s^{-1}$. The numerical values of the other rate constants are given in the captions of the figures 4, 5] and 6 .

Unlike the cytoskeletal motors, a single RNAP is so large that it can simultaneously cover $r$ successive nucleotides on the track. In our terminology, a site is occupied by a RNAP if it coincides with the leftmost of the $r$ sites representing that RNAP while the next $r-1$ sites on its right are said to be covered by the same RNAP. Irrespective of the actual numerical value of $r$, each RNAP can move forward or backward by only one site in each time step, if demanded by its own mechano-chemistry, provided the target site is not already covered by any other RNAP. The total number of RNAPs on the DNA template is denoted by the symbol $N$. Thus, $\rho=N / L$ is the number density of the RNAPs. The coverage density is defined by $\rho_{\text {cov }}=N r / L$ which is the total fraction of the nucleotides covered by all the RNAPs together.

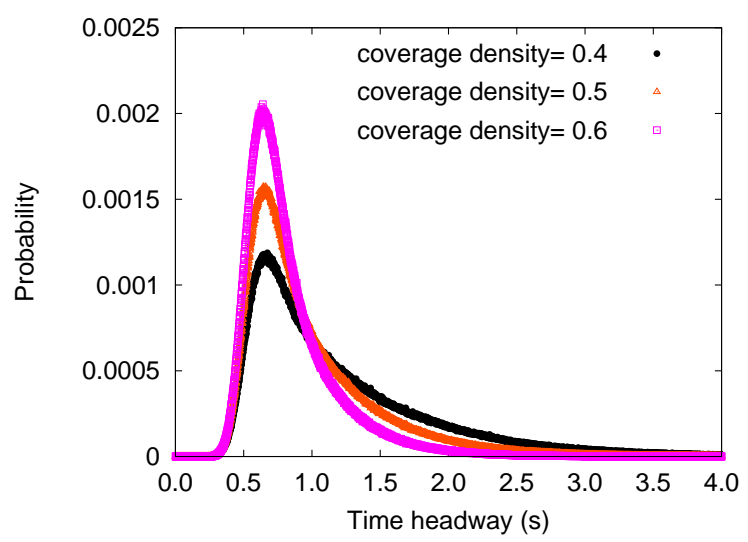

FIG. 4: Distributions of the time-headways in the model of RNAP traffic under PBC. The three curves correspond to three different coverage densities of the RNAP motors, all for fixed $\omega_{21}^{f}=100$ and $\omega_{21}=1.0$.

Let $P_{\mu}(i, t)$ denote the probability that there is a RNAP at the spatial position $i$ and in the chemical state $\mu$ at time $t$. Let $Q(\underline{i} \mid j)$ be the conditional probability that, given a RNAP at site $i$, site $j$ is empty. Note that, if site $i$ is given to be occupied by one RNAP, the site $i-1$ can be covered by another RNAP if, and only if, the site $i-r$ is also occupied. In the mean-field approximation, the master equations for $P_{\mu}(i, t)$ are given by [16]

$$
\begin{aligned}
\frac{d P_{1}(i, t)}{d t} & =\omega_{11}^{f} P_{1}(i-1, t) Q(\underline{i-1} \mid i-1+r) \\
& +\omega_{11}^{b} P_{1}(i+1, t) Q(i+1-r \mid \underline{i+1}) \\
& +\omega_{12}^{b} P_{2}(i+1, t) Q(i+1-r \mid \underline{i+1}) \\
& +\omega_{12} P_{2}(i, t)-\omega_{21} P_{1}(i, t) \\
& -\left(\omega_{11}^{f}+\omega_{21}^{f}\right) P_{1}(i, t) Q(\underline{i} \mid i+r) \\
& -\omega_{11}^{b} P_{1}(i, t) Q(i-r \mid \underline{i})
\end{aligned}
$$

$$
\begin{aligned}
\frac{d P_{2}(i, t)}{d t} & =\omega_{22}^{f} P_{2}(i-1, t) Q(\underline{i-1} \mid i-1+r) \\
& +\omega_{22}^{b} P_{2}(i+1, t) Q(i+1-r \mid \underline{i+1}) \\
& +\omega_{21}^{f} P_{1}(i-1, t) Q(\underline{i-1} \mid i-1+r) \\
& +\omega_{21} P_{1}(i, t)-\omega_{12} P_{2}(i, t) \\
& -\left(\omega_{22}^{b}+\omega_{12}^{b}\right) P_{2}(i, t) Q(i-r \mid \underline{i}) \\
& -\omega_{22}^{f} P_{2}(i, t) Q(\underline{i} \mid i+r)
\end{aligned}
$$


In the steady state under PBC,

$$
\begin{aligned}
& P_{1}=\left(\frac{\omega_{12}+\omega_{12}^{b} Q}{\Omega_{\uparrow}+\Omega_{\leftrightarrow} Q}\right) \rho \\
& P_{2}=\left(\frac{\omega_{21}+\omega_{21}^{f} Q}{\Omega_{\uparrow}+\Omega_{\leftrightarrow} Q}\right) \rho
\end{aligned}
$$

where

$$
\begin{gathered}
\Omega_{\uparrow}=\omega_{12}+\omega_{21} \\
\Omega_{\leftrightarrow}=\omega_{21}^{f}+\omega_{12}^{b}
\end{gathered}
$$

and $Q$ is given by

$$
Q(\underline{i} \mid i+r)=Q(i \mid \underline{i+r})=\frac{1-\rho r}{1+\rho-\rho r}
$$

The corresponding steady-state flux is given by

$$
\begin{aligned}
J & =\Omega_{1} P_{1} Q+\Omega_{2} P_{2} Q \\
& =\left(\Omega_{1} P_{1}+\Omega_{2} P_{2}\right)\left(\frac{1-\rho_{\text {cov }}}{1+\rho-\rho_{\text {cov }}}\right)
\end{aligned}
$$

where

$$
\begin{gathered}
\Omega_{1}=\omega_{11}^{f}+\omega_{21}^{f}-\omega_{11}^{b} \\
\Omega_{2}=\omega_{22}^{f}-\omega_{12}^{b}-\omega_{22}^{b},
\end{gathered}
$$

are two effective forward hopping rates from the states 1 and 2 , respectively.

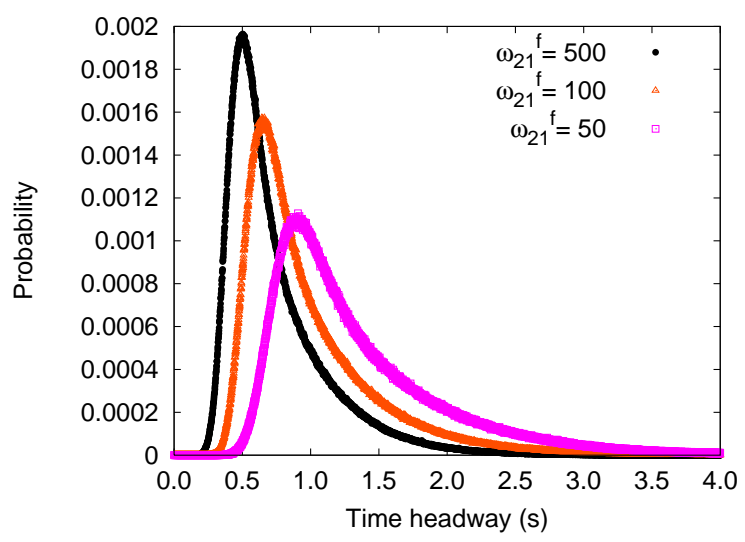

FIG. 5: Distributions of the time-headways in the model of RNAP traffic under PBC. Different curves correspond to different values of $\omega_{21}^{f}$, all for the fixed coverage density 0.5 and $\omega_{21}=1.0$.

Since, unlike vehicles in highway traffic, an RNAP motor can step backward, TH under PBC must be computed carefully as follows [45]: we label the motors sequentially in the beginning. Because of the PBC and because of the impossibility of overtaking, the sequence of the labels remain unchanged during the time evolution of the system. $\mathrm{TH}$ is defined as the time interval between the departure (i.e., forward hopping) of the successive motors from the same site.

Typical TH distributions of the RNAPs in this model under PBC are plotted in figs 4, 5, 6 for biologically relevant sets of values of the parameters [45]; the corresponding results under OBC have been reported elsewhere [16]. In these parameter regimes, because of the "chemical transition" between the states 1 and 2, the minimum TH is non-zero, i.e., $P(\tau)=0$ for all $\tau \leq \tau_{\text {min }}$. The magnitude of $\tau_{\min }$ as well as the most probable $\mathrm{TH}$ are practically independent of the coverage density. Moreover, both $\tau_{\min }$ and the most probable $\mathrm{TH}$ decrease with increasing $\omega_{21}^{f}$, (i.e., with increasing concentration of the monomeric subunits of the growing mRNA). In contrast, $\tau_{\text {min }}$ and the most probable $\mathrm{TH}$ increase with increasing $\omega_{21}$ which tends to suppress forward movement of each RNAP along the main pathway (see fig 3). The trends of variation of the most probable $\mathrm{TH}$ with the model parameters are consistent with the corresponding trends of variation of the average flux with the same parameters.

Very recently we have proposed [16] that the width of the TH distribution, as defined above in the context of RNAP traffic, can serve as a good quantitative measure of noise in transcription. Recent progress in imaging techniques has made it possible to monitor the synthesis of successive individual RNAs in a living cell [46]. Using these techniques it has been demonstrated [47, 48, 49] that synthesis of RNAs take place in "bursts" [50]. The time series of the events correspoding to the $\mathrm{TH}$ distributions shown in figs 4, 5 and 6 do not account for such "bursts". But "bursting" is observed when our model is extended by incorporating the processes of "swtiching on" and "switching off" of the gene [51].

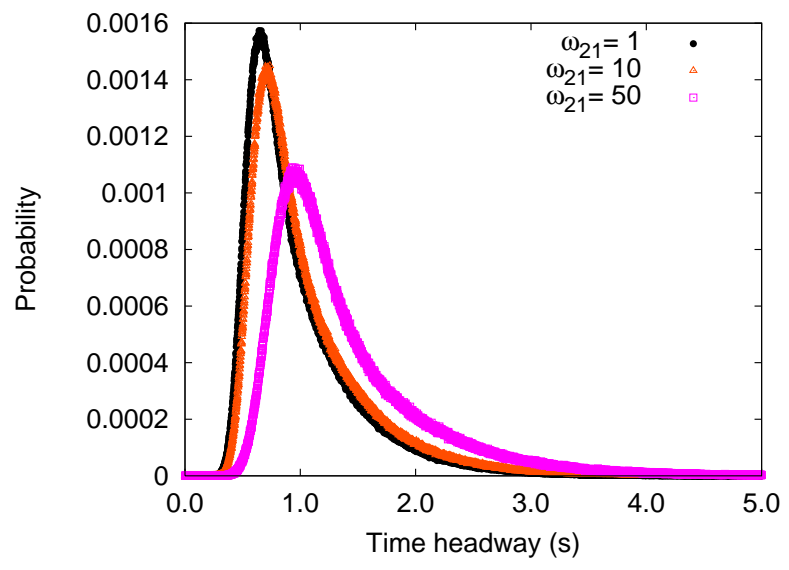

FIG. 6: Distributions of the time-headways in the model of RNAP traffic under PBC. Different curves correspond to different values of $\omega_{21}$, all for the fixed coverage density 0.5 and $\omega_{21}^{f}=100$. 


\section{TRAFFIC OF RIBOSOMES ON MRNA TRACKS}

Synthesis of a protein from the corresponding messenger RNA (mRNA) is carried out by a motor called ribosome [52] and the process is referred to as translation of genetic code. Usually, many ribosomes move simultaneously on a single mRNA strand while each synthesizes a separate copy of the same protein. Strictly speaking, a ribosome is neither a particle nor a hard rod $[53,54,55,56,57,58,59,60,61]$; its mechanical movement along the mRNA track is coupled to its internal mechanochemical processes which also drive the synthesis of the protein.

Very recently, we have developed a model that not only incorporates the inter-ribosome steric interactions, but also captures explicitly the essential steps in the intraribosome chemomechanical processes. This model for ribosome traffic is very similar to that for RNAP traffic except that (i) each lattice represents a codon (a triplet of nucleotides) and (ii) the mechano-chemical of a single ribosome (see fig [7) is quite different from that of a RNAP.

Each ribosome consists of two subunits. The smaller subunit binds to the mRNA track. But, the actual elongation of the protein takes place in the larger subunit by the addition of the successive monomers, called amino acid. The operations of the two subunits are coordinated by an adaptor molecule called tRNA. It uses the anticodon at one of its ends to decode the genetic instructions stored in each codon of the mRNA which serves also as the template for synthesis of a protein. Each tRNA carries an amino acid at its other end. Correct codonanticodon matching ensures that the correct amino acid, as dictated by the mRNA template, is used by the larger subunit for elongating the protein by one monomer.

The main steps in the mechano-chemical cycle of a ribosome are the following:

(i) arrival of the correct tRNA (rate constant $\omega_{a}$ ), (ii) growth of the protein by one monomer because of the formation of a covalent bond with the newly arrived amino acid (rate $\omega_{g}$ ), and (iii) forward movement of the ribosome by one codon which is associated with the hydrolysis of a guanosine triphosphate (GTP) molecule (rate $\left.\omega_{h 2}\right)$. The rate constants $\omega_{h 1}$ and $k_{2}$ are associated with two steps during which another GTP molecule is hydrolyzed. A more detailed description of the model is available in ref.[17]. The biologically relevant values of the parameters, which have been kept fixed throughout our studies of the TH distributions, are as follows: $\omega_{p}=0.0028 s^{-1}, k_{2}=2.4 s^{-1}$ and $\omega_{g}=2.5 s^{-1}$.

Under mean-field approximation, the master equations for the probabilities $P_{\mu}(i)$ are given by [17]

$\frac{\partial P_{1}(i)}{\partial t}=\omega_{h 2} P_{5}(i-1) Q(\underline{\mathrm{i}-1} \mid i-1+r)+\omega_{p} P_{2}(i)-\omega_{a} P_{1}(i)$

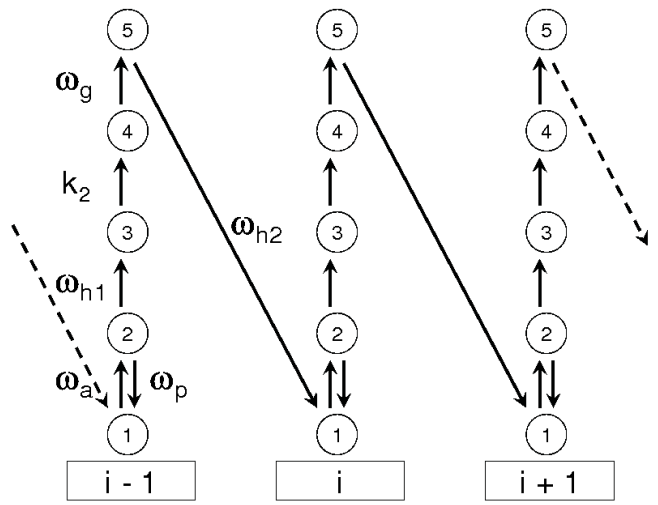

FIG. 7: A schematic representation of the biochemical cycle of a single ribosome during the elongation stage of translation in our model [17]. The index below the box labels the codon on the mRNA with which the ribosome binds. Each circle labelled by an integer index represents a distinct state in the mechano-chemical state of a ribosome. The symbols accompanied by the arrows define the rate constants for the corresponding transitions from one state to another.

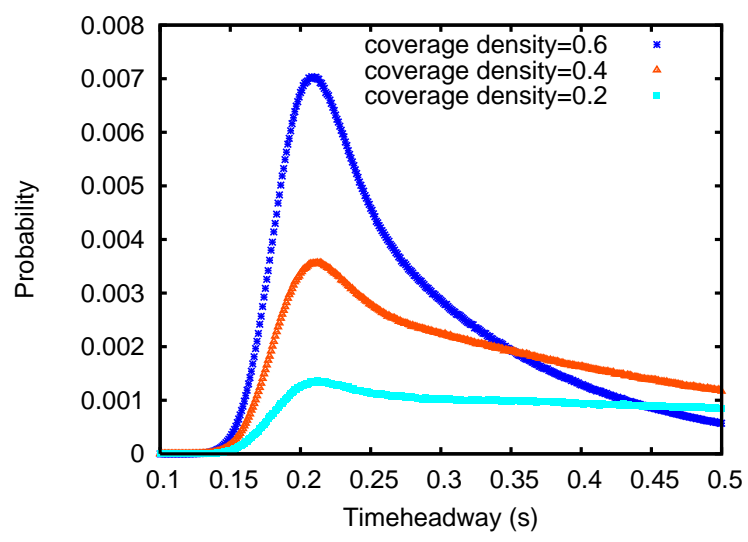

FIG. 8: TH distributions in the model of ribosome traffic under PBC. The three curves correspond to three different coverage densities of the ribosomes, all for $\omega_{a}=2.5 \mathrm{~s}^{-1}$ and $\omega_{h 1}=\omega_{h 2}=\omega_{h}=10 s^{-1}$.

$$
\begin{gathered}
\frac{\partial P_{2}(i)}{\partial t}=\omega_{a} P_{1}(i)-\left(\omega_{p}+\omega_{h 1}\right) P_{2}(i) \\
\frac{\partial P_{3}(i)}{\partial t}=\omega_{h 1} P_{2}(i)-k_{2} P_{3}(i) \\
\frac{\partial P_{4}(i)}{\partial t}=k_{2} P_{3}(i)-\omega_{g} P_{4}(i) \\
\frac{\partial P_{5}(i)}{\partial t}=\omega_{g} P_{4}(i)-\omega_{h 2} P_{5}(i) Q(\underline{\mathrm{i}} \mid i+r)
\end{gathered}
$$




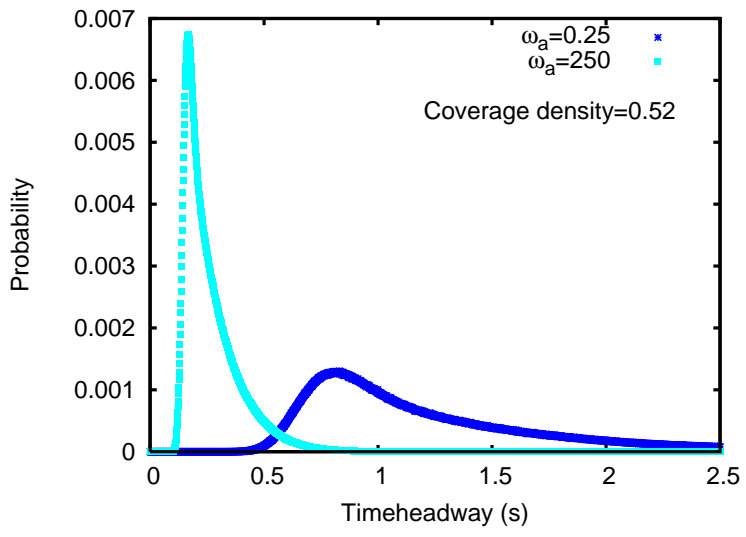

FIG. 9: TH distributions in the model of ribosome traffic under PBC. Different curves correspond to different values of $\omega_{a}$, all for coverage density $=0.52$ and $\omega_{h 1}=\omega_{h 2}=\omega_{h}=$ $10 s^{-1}$.

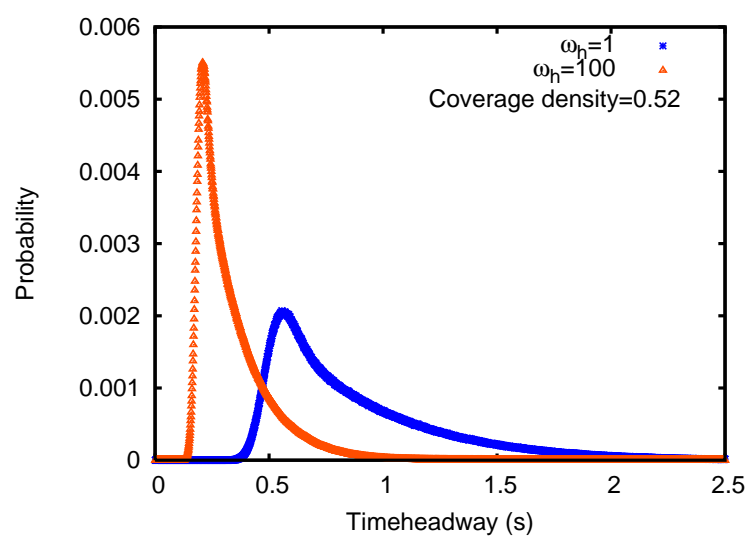

FIG. 10: TH distributions in the model of ribosome traffic under PBC. Different curves correspond to different values of $\omega_{h}$, all for coverage density $=0.52$ and $\omega_{a}=2.5 s^{-1}$.

In the steady state under PBC, the flux of the ribosomes is given by

$$
J=\frac{\omega_{h 2} \rho(1-\rho r)}{(1+\rho-\rho r)+\Omega_{h 2}(1-\rho r)}
$$

where

$$
\Omega_{h 2}=\omega_{h 2} / k_{e f f}
$$

with

$$
\frac{1}{k_{e f f}}=\frac{1}{\omega_{g}}+\frac{1}{k_{2}}+\frac{1}{\omega_{h 1}}+\frac{1}{\omega_{a}}+\frac{\omega_{p}}{\omega_{a} \omega_{h 1}}
$$

$\mathrm{TH}$ distributions of the ribosomes in this model under $\mathrm{PBC}$ are plotted in figs 8, 9, 10 for different sets of parameters [26]; the corresponding results under OBC have been reported elsewhere [19]. The qualitative features of the $\mathrm{TH}$ distributions and their trend of variation with the parameters of the model of ribosome traffic are very similar to those observed in the preceeding section in the context of RNAP traffic. Neither $\tau_{\min }$ nor the most probable TH depend on the coverage density. But, both $\tau_{\min }$ and most probable TH decrease with increasing $\omega_{a}$ which is a measure of the abundance of the amino acid subunits of the growing protein chain. A similar trend of variation of TH distribution is also observed with increasing $\omega_{h}$ which is a measure of the rate of "fuel" consumption (more precisely, rate of GTP hydrolysis).

The width of the $\mathrm{TH}$ distribution in ribosome traffic can serve as a mesure of translational noise [19], just as that in RNAP traffic has been considered in section III and in ref. [16] as a measure of transcriptional noise. A comparison between the trend of variation of this noise with our model parameters and the corresponding recent experimental observations has been reported elsewhere [19].

\section{DH DISTRIBUTIONS IN MOLECULAR MOTOR TRAFFIC}

Because of the possibilities of attachments and detachments of the motors, the DH distribution is not a suitable quantity for characterizing KIF1A motor traffic. Therefore, in this section, we shall consider exclusively only traffic of RNAPs and ribosomes under PBC; the corresponding results under OBC are discussed in ref. [45] and ref. [26].

The DH distribution in the TASEP with hard rods, each of length $r$, was calculated analytically by Shaw et al. [56]. Suppose, $m$ denotes the DH. The DH-distribution $P(m)$ in this model under $\mathrm{PBC}$ is given by 56 .

$$
P(m)=\left(\frac{\rho}{\rho_{s}}\right)\left(\frac{\rho_{h}}{\rho_{s}}\right)^{m}
$$

where $\rho_{h}=1-\rho_{\text {cov }}$ is the fraction of the system covered by the holes, and $\rho_{s}=\rho+\rho_{h}$. In the limit $r=1, \rho_{\text {cov }}=\rho$, $\rho_{s}=1$ and, hence, $P(m)$ reduces to the limiting form $P(m)=\rho(1-\rho)^{m}$ which is the well-known mean-field estimate of the DH distribution for TASEP [12, 13].

In fig 11 we have plotted the $\mathrm{DH}$ distributions in the models of RNAP traffic and ribosome traffic under PBC where $r=10$ for both the models. Interestingly, for any given coverage density, the $\mathrm{DH}$ distributions in both these models follow equation (21) in spite of the differences in the mechano-chemical cycle of the individual motors. Thus, the DH distributions in these models of molecular motor traffic are determined solely by the geometric parameters.

\section{SUMMARY AND CONCLUSION}

In this paper we have modelled molecular motor transport on filamentary tracks from the perspective of physi- 


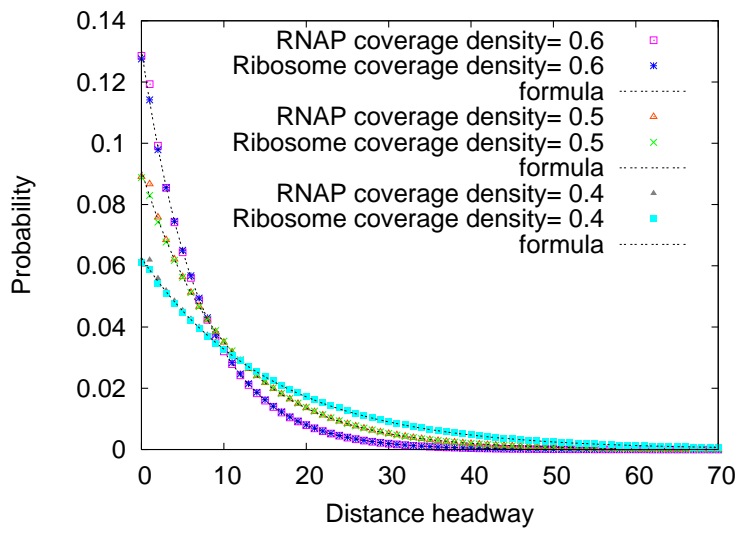

FIG. 11: DH distribution in RNAP traffic and ribosome traffic.

cists and traffic scientists. These models are essentially biologically motivated extensions of TASEP. To our knowledge, TASEP is the simplest model of non- equilibrium systems of interacting self-driven particles. Moreover, many extensions of TASEP have been used earlier to capture various phenomena observed in vehicular traffic. The interesting quantities which are used for characterizing traffic flow include fundamental diagram, distributions of distance- and time-headways. In this paper, for all the models of molecular motor traffic, we have not only summarized the earlier results on the fundamental diagrams, but also presented many new results on the distributions of $\mathrm{DH}$ and $\mathrm{TH}$. The $\mathrm{TH}$ distribution in traffic of RNAPs and ribosomes yield novel measures of intrinsic noise in transcription and translation, respectively [16, 19]. Our modeling provide new insight into intracellular motor-driven processes by looking at these from a novel perspective.

Acknowledgements: It is our great pleasure to thank all our collaborators for enjoyable collaborations. This work is supported (through DC) by a research grant from CSIR (India).
[1] J. Howard, Mechanics of Motor Proteins and the Cytoskeleton, (Sinauer Associates, 2001) .

[2] M. Schliwa (ed.), Molecular Motors, (Wiley-VCH, 2002).

[3] A.B. Kolomeisky and M.E. Fisher, in: Annual Review of Physical Chemistry, 58, 675 (2007).

[4] D.D. Hackney and F. Tamanoi, The Enzymes, vol.XXIII, Energy Coupling and Molecular Motors (Elsevier, 2004).

[5] D. Chowdhury, A. Schadschneider and K. Nishinari, Phys. of Life Rev. 2, 318 (2005).

[6] Special issue 1 of vol. $\mathbf{3 7 2}$ of Physica A, on "common trends in traffic systems" guest eds. D. Chowdhury, B. K. Chakrabarti and A. Dutta (2006).

[7] G. M. Schütz, in: Phase Transitions and Critical Phenomena, vol. 19 (Acad. Press, 2001).

[8] J. Krug, Phys. Rev. Lett. 67, 1882 (1991)

[9] D. Chowdhury, L. Santen, and A. Schadschneider, Phys. Rep. 329, 199 (2000).

[10] K. Ghosh, A. Majumdar and D. Chowdhury, Phys. Rev. E 58, 4012 (1998).

[11] D. Chowdhury, A. Pasupathy and S. Sinha, Eur. Phys. J. B 5, 781 (1998).

[12] D. Chowdhury, K. Ghosh, A. Majumdar, S. Sinha and R.B. Stinchcombe, Physica A, 246, 471 (1997).

[13] A. Schadschneider and M. Schreckenberg, J. Phys. A 30, L69 (1997).

[14] K. Nishinari, Y. Okada, A. Schadschneider and D. Chowdhury, Phys. Rev. Lett. 95, 118101 (2005).

[15] P. Greulich, A. Garai, K. Nishinari, A. Schadschneider and D. Chowdhury, Phys. Rev. E 75, 041905 (2007).

[16] T. Tripathi and D. Chowdhury, Phys. Rev. E (2008) (in press).

[17] A. Basu and D. Chowdhury, Phys. Rev. E 75, 021902 (2007)

[18] A. Basu and D. Chowdhury, Amer. J. Phys. 75, 931 (2007).

[19] A. Garai, D. Chowdhury and T.V. Ramakrishnan (to be published, 2007).

[20] R. Lipowsky, S. Klumpp and T.M. Nieuwenhuizen, Phys. Rev. Lett. 87, 108101 (2001).

[21] R. Lipowsky, Y. Chai, S. Klumpp, S. Liepelt and M. J.I. Müller, Physica A 372, 34 (2006) and references therein.

[22] A. Parmeggiani, T. Franosch and E. Frey, Phys. Rev. Lett. 90, 086601 (2003); Phys. Rev. E 70, 046101 (2004).

[23] E. Frey, A. Parmeggiani and T. Franosch, Genome Inf. 15, 46 (2004) and references therein.

[24] M.R. Evans, R. Juhasz and L. Santen, Phys. Rev. E 68, 026117 (2003).

[25] V. Popkov, A. Rakos, R.D. Williams, A.B. Kolomeisky and G.M. Schütz, Phys. Rev. E 67, 066117 (2003).

[26] A. Garai, Ph.D. Thesis, IIT Kanpur (in preparation).

[27] L. Bai, T.J. Santangelo and M.D. Wang, Annu. Rev. Biophys. Biomol. Str. 35, 343 (2006).

[28] F. Jülicher and R. Bruinsma, Biophys. J. 74, 1169 (1998).

[29] H.Y. Wang, T. Elston, A. Mogilner and G. Oster, Biophys. J. 74, 1186 (1998).

[30] R. Sousa, Trends in Biochem. Sci. 21, 186 (1996).

[31] R. Guajardo and R. Sousa, J. Mol. Biol. 265, 8 (1997).

[32] Q. Guo and R. Sousa, J. Mol. Biol. 358, 241 (2006).

[33] L. Bai, A. Shundrovsky and M.D. Wang, J. Mol. Biol. 344, 335 (2004).

[34] L. Bai, R.M. Fulbright and M.D. Wang, Phys. Rev. Lett. 98, 068103 (2007).

[35] G. Bar-Nahum, V. Epshtein, A.E. Ruckenstein, R. Rafikov, A. Mustaev and E. Nudler, Cell 120, 183 (2005).

[36] V.R. Tadigotla, D.O. Maoileidigh, A.M. Sengupta, V. Epshtein, R.H. Ebright, E. Nudler and A.E. Ruckenstein, Proc. Nat. Acad. Sci. USA, 103, 4439 (2006).

[37] Y. R. Yamada and C.S. Peskin, arxiv:q-bio.BM/0603012 (2006).

[38] H.J. Woo, Phys. Rev. E 74, 011907 (2006).

[39] V. Epshtein and E. Nudler, Science 300, 801 (2003).

[40] V. Epshtein, F. Toulme, A. Rachid Rahmouni, S. 
Borukhov and E. Nudler, EMBO J. 22, 4719 (2003).

[41] N. Crampton, W.A. Bonass, J. Kirkham, C. Rivetti and N.H. Thomson, Nucleic Acids Research, 34, 5416 (2006).

[42] K. Sneppen, I.B. Dodd, K.E. Shearwin, A.C. Palmer, R.A. Schubert, B.P. Callen and J.B. Egan, J. Mol. Biol. 346, 399 (2005).

[43] H. Bremer and M. Ehrenberg, Biochim. Biophys. Acta 1262, 15 (1995).

[44] K.E. Shearwin, B.P. Callen and J.B. Egan, Trends in Genetics 21, 339 (2005).

[45] T. Tripathy, Ph.D. Thesis, IIT Kanpur (in preparation).

[46] Y. Shav-Tal, R.H. Singer and X. Darzacq, Nat. Rev. Mol. Cell Biol. 5, 856 (2004).

[47] I. Golding, J. Paulsson, S.M. Zawilski and E.C. Cox, Cell 123, 1025 (2005).

[48] J.R. Chubb, T. Trcek, S.M. Shenoy and R.H. Singer, Curr. Biol. 16, 1018 (2006).

[49] A. Raj, C.S. Peskin, D. Tranchina, D.Y. Vargas and S. Tyagi, PloS Biol. 4, 1707 (2006).
[50] I. Golding and E.C. Cox, Curr. Biol. 16, R371 (2006).

[51] T. Tripathy and D. Chowdhury, to be published.

[52] A. S. Spirin, Ribosomes, (Springer, 2000).

[53] C. MacDonald, J. Gibbs and A. Pipkin, Biopolymers, 6, 1 (1968).

[54] C. MacDonald and J. Gibbs, Biopolymers, 7, 707 (1969).

[55] G. Lakatos and T. Chou, J. Phys. A 36, 2027 (2003).

[56] L.B. Shaw, R.K.P. Zia and K.H. Lee, Phys. Rev. E 68, 021910 (2003).

[57] L.B. Shaw, J.P. Sethna and K.H. Lee, Phys. Rev. E 70, 021901 (2004).

[58] L.B. Shaw, A.B. Kolomeisky and K.H. Lee, J. Phys. A 37, 2105 (2004).

[59] T. Chou, Biophys. J., 85, 755 (2003).

[60] T. Chou and G. Lakatos, Phys. Rev. Lett. 93, 198101 (2004).

[61] J.J. Dong, B. Schmittmann and R.K.P. Zia, J. Stat. Phys. 128, 21 (2007). 